\title{
Hg(II)-Selective Excimer Emission of a Bis-Naphthyl Azadiene Derivative
}

\author{
Yasuhiro Shiraishi,* Hajime Maehara, Katsutake Ishizumi, and Takayuki Hirai \\ Research Center for Solar Energy Chemistry, and Division of Chemical Engineering, \\ Graduate School of Engineering Science, Osaka University, Toyonaka 560-8531, Japan \\ E-mail: shiraish@cheng.es.osaka-u.ac.jp.
}

\section{Supporting Information}

\section{Experimental Procedure.}

Solvents and raw materials were of the highest commercial quality, supplied from Aldrich, Wako, and Tokyo Kasei and used without further purification. Water was purified by the Milli Q system. Steady-state fluorescence spectra were measured on a Hitachi F-4500 fluorescence spectrophotometer $\left(\lambda_{\mathrm{exc}}=372 \mathrm{~nm} ; \lambda_{\mathrm{em}}=492 \mathrm{~nm}\right.$, excitation and emission slit width, $\left.2.5 \mathrm{~nm}\right)$. Absorption spectra were measured on an UV-visible photodiode-array spectrophotometer (Shimadzu; Multispec-1500). Nanosecond time-resolved fluorescence decay measurements were carried out on a PTI-3000 apparatus (Photon Technology International) using a Xe nanoflash lamp filled with $\mathrm{N}_{2}$ as the light excitation source $\left(\lambda_{\text {exc }}=358 \mathrm{~nm} ; \lambda_{\text {em }}=480 \mathrm{~nm}\right)$. All spectroscopic measurements were carried out at $298 \pm 0.1 \mathrm{~K}$ using a $10 \mathrm{~mm}$ path length quartz cell. For reproduction of the data, all spectral measurements were carried out in deaerated acetonitrile under dry $\mathrm{N}_{2}$ atmosphere. ${ }^{1} \mathrm{H}$ and ${ }^{13} \mathrm{C}$ NMR spectra were recorded by a JEOL JNM-GSX270 Excalibur. FAB-MS spectra were obtained by a JEOL JMS 700 Mass Spectrometer. Fluorescence quantum yields were determined based on anthracene as standard (in ethanol, $\Phi=0.27 \pm 0.01$ ), ${ }^{1}$ using the equation $\Phi_{\mathrm{x}} / \Phi_{\mathrm{s}}=\left(\mathrm{S}_{\mathrm{x}} / \mathrm{S}_{\mathrm{s}}\right)\left[\left(1-10^{-\mathrm{As}}\right) /\left(1-10^{-\mathrm{Ax}}\right)\right]{ }^{2}$, where $\mathrm{x}$ and $\mathrm{s}$ indicate the unknown and standard solution, respectively, $\Phi$ is the quantum yield, $\mathrm{S}$ is the area under the emission curve and $\mathrm{A}$ is the absorbance at the excitation wavelength $\left(\lambda_{\text {exc }}=365 \mathrm{~nm}\right)$.

\section{Calculation Details.}

Preliminary Geometry optimizations of metal complexes were performed using WinMOPAC version 3.0 software (Fujitsu Inc.) at the semiempirical MNDO-d level. Geometry of the complexes was optimized employing $\mathrm{Zn}^{2+}$ instead of $\mathrm{Hg}^{2+}$. The obtained structures were then fully refined in the gas-phase with tight convergence criteria at the DFT level with the Gaussian 03 package, ${ }^{3}$ with 
replacing $\mathrm{Zn}^{2+}$ with $\mathrm{Hg}^{2+}$, using the $\mathrm{B}^{2} \mathrm{LYP}^{4}$ functional and the 6-31G* (for 2:1 complex) or 3-21G (for 2:2 and 2:3 complexes) basis set for all atoms except $\mathrm{Hg}$, for which the Stuttgart Relativistic Small-Core basis $\operatorname{set}^{5}((8 \mathrm{~s} 8 \mathrm{p} 7 \mathrm{~d}) \rightarrow[6 \mathrm{~s} 6 \mathrm{p} 4 \mathrm{~d}]$ contraction $)$ with effective core potential was used. The Cartesian coordinates for respective $2: 1,2: 2$, and $2: 3 \mathbf{L}-\mathrm{Hg}^{2+}$ complexes are summarized in the end of Supporting Information.

[1] Melhuish, W. H. J. Phys. Chem. 1961, 65, 229.

[2] Valeur, B. Molecular Fluorescence: Principles and Applications, Wiley-VCH, New York, 2001.

[3] Gaussian 03, Revision B.03, Frisch, M. J.; Trucks, G. W.; Schlegel, H. B.; Scuseria, G. E.; Robb, M. A.; Cheeseman, J. R.; Montgomery, Jr., J. A.; Vreven, T.; Kudin, K. N.; Burant, J. C.; Millam, J. M.; Iyengar, S. S.; Tomasi, J.; Barone, V.; Mennucci, B.; Cossi, M.; Scalmani, G.; Rega, N.; Petersson, G. A.; Nakatsuji, H.; Hada, M.; Ehara, M.; Toyota, K.; Fukuda, R.; Hasegawa, J.; Ishida, M.; Nakajima, T.; Honda, Y.; Kitao, O.; Nakai, H.; Klene, M.; Li, X.; Knox, J. E.; Hratchian, H. P.; Cross, J. B.; Bakken, V.; Adamo, C.; Jaramillo, J.; Gomperts, R.; Stratmann, R. E.; Yazyev, O.; Austin, A. J.; Cammi, R.; Pomelli, C.; Ochterski, J. W.; Ayala, P. Y.; Morokuma, K.; Voth, G. A.; Salvador, P.; Dannenberg, J. J.; Zakrzewski, V. G.; Dapprich, S.; Daniels, A. D.; Strain, M. C.; Farkas, O.; Malick, D. K.; Rabuck, A. D.; Raghavachari, K.; Foresman, J. B.; Ortiz, J. V.; Cui, Q.; Baboul, A. G.; Clifford, S.; Cioslowski, J.; Stefanov, B. B.; Liu, G.; Liashenko, A.; Piskorz, P.; Komaromi, I.; Martin, R. L.; Fox, D. J.; Keith, T.; Al-Laham, M. A.; Peng, C. Y.; Nanayakkara, A.; Challacombe, M.; Gill, P. M. W.; Johnson, B.; Chen, W.; Wong, M. W.; Gonzalez, C.; and Pople, J. A.; Gaussian, Inc., Wallingford CT, 2004.

[4] Bartolottiand, L.J.; Fluchick, K. Reviews in Computational Chemistry, VCH, New York, 1996; Vol. 7, pp. 187-216.

[5] The basis set was obtained from the Extensible Computational Chemistry Environment Basis Set Database, Version 02/25/04, as developed and distributed by the Molecular Science Computing Facility, Environmental and Molecular Sciences Laboratory which is part of the Pacific Northwest Laboratory, P.O. Box 999, Richland, Washington 99352, USA, and funded by the U.S. Department of Energy. The Pacific Northwest Laboratory is a multi-program laboratory operated by Battelle Memorial Institute for the U.S. Department of Energy under contract DE-AC06-76RLO 1830. 

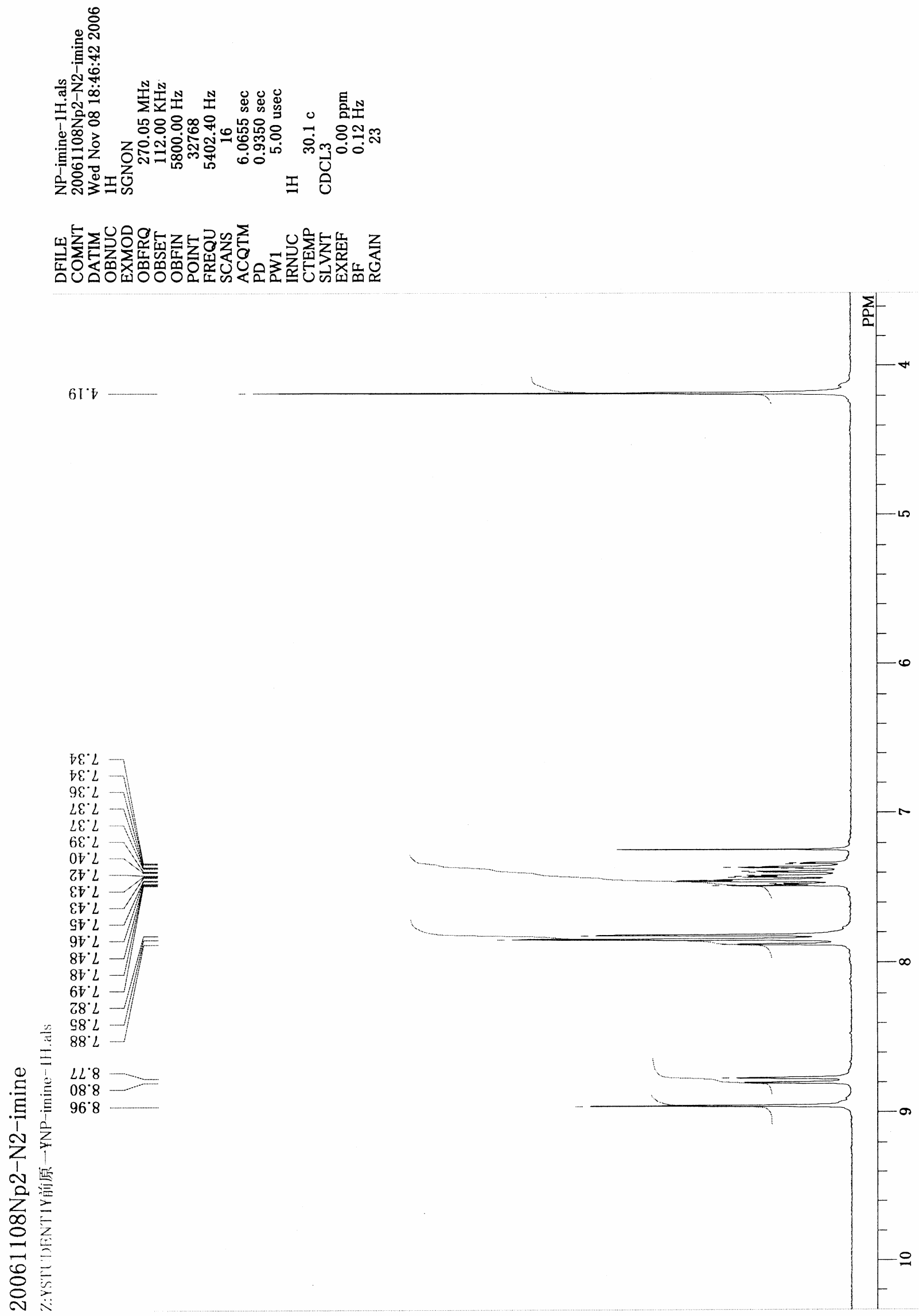

Figure S1. ${ }^{1} \mathrm{H}$ NMR spectrum of $\mathbf{L}\left(\mathrm{CDCl}_{3}, 270 \mathrm{MHz}\right)$ 

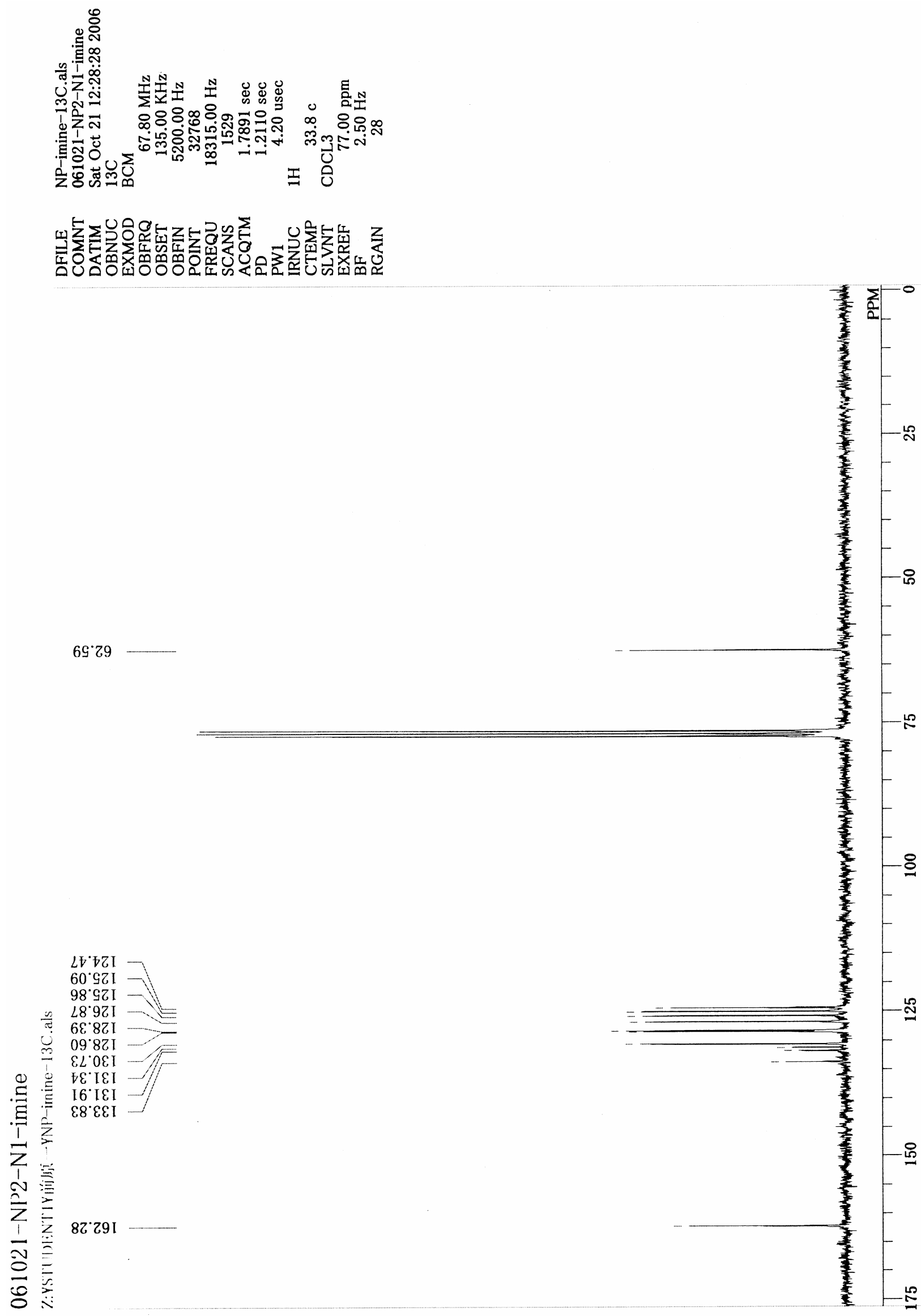

Figure S2. ${ }^{13} \mathrm{C}$ NMR spectrum of $\mathbf{L}\left(\mathrm{CDCl}_{3}, 68 \mathrm{MHz}\right)$ 


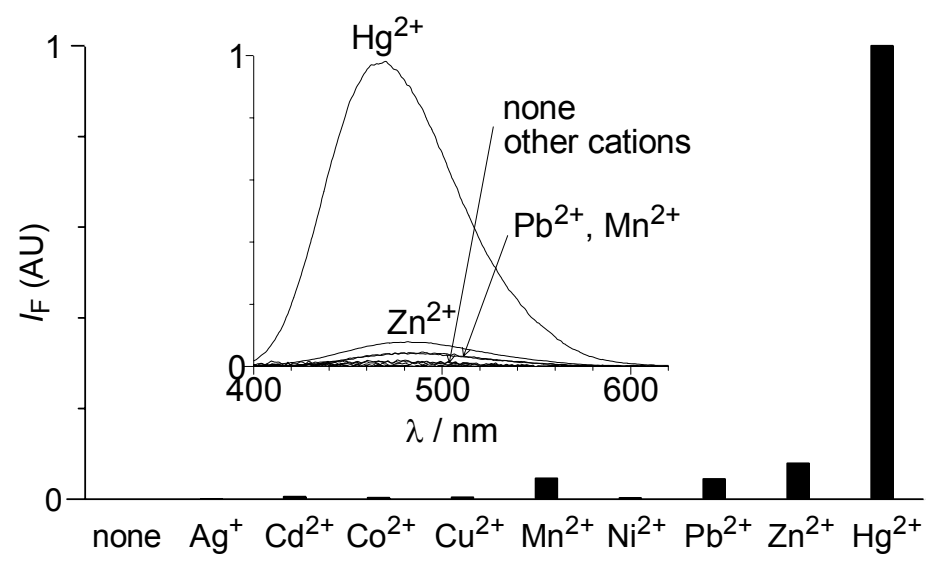

Figure S3. Emission spectra $\left(\lambda_{\text {exc }}=372 \mathrm{~nm}\right)$ of $\mathbf{L}(25 \mu \mathrm{M})$ and intensity at $492 \mathrm{~nm}$ measured in acetonitrile with and without 5 equiv of respective metal cations as perchlorate salts.
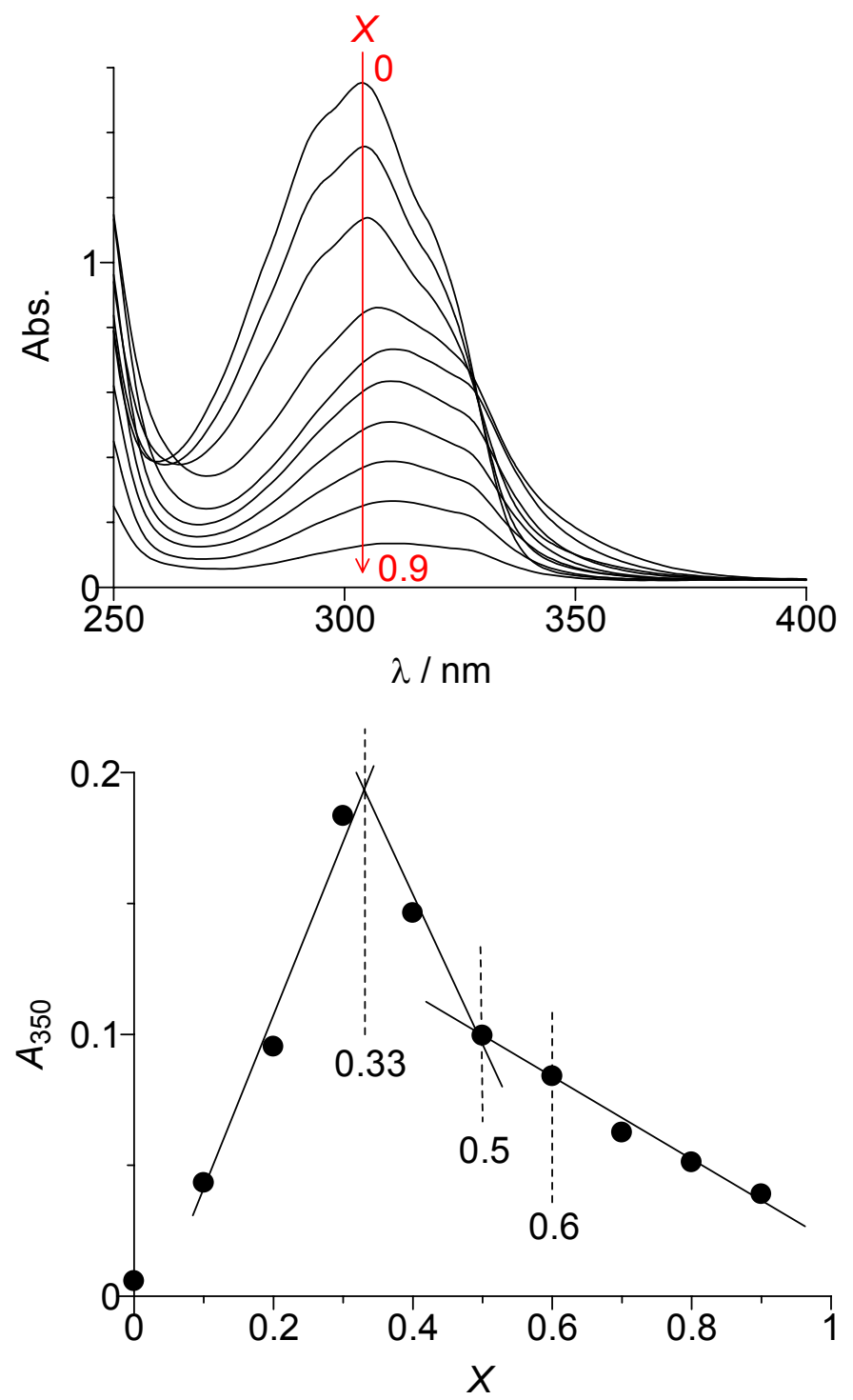

Figure S4. Job's plot of $\mathbf{L}$ with $\mathrm{Hg}^{2+}$ obtained by absorption measurements $\left(X=\left[\mathrm{Hg}^{2+}\right] /\left(\left[\mathrm{Hg}^{2+}\right]+\right.\right.$ $[\mathbf{L}])$. The total concentration of $\mathbf{L}$ and $\mathrm{Hg}^{2+}$ is $100 \mu \mathrm{M}$. 

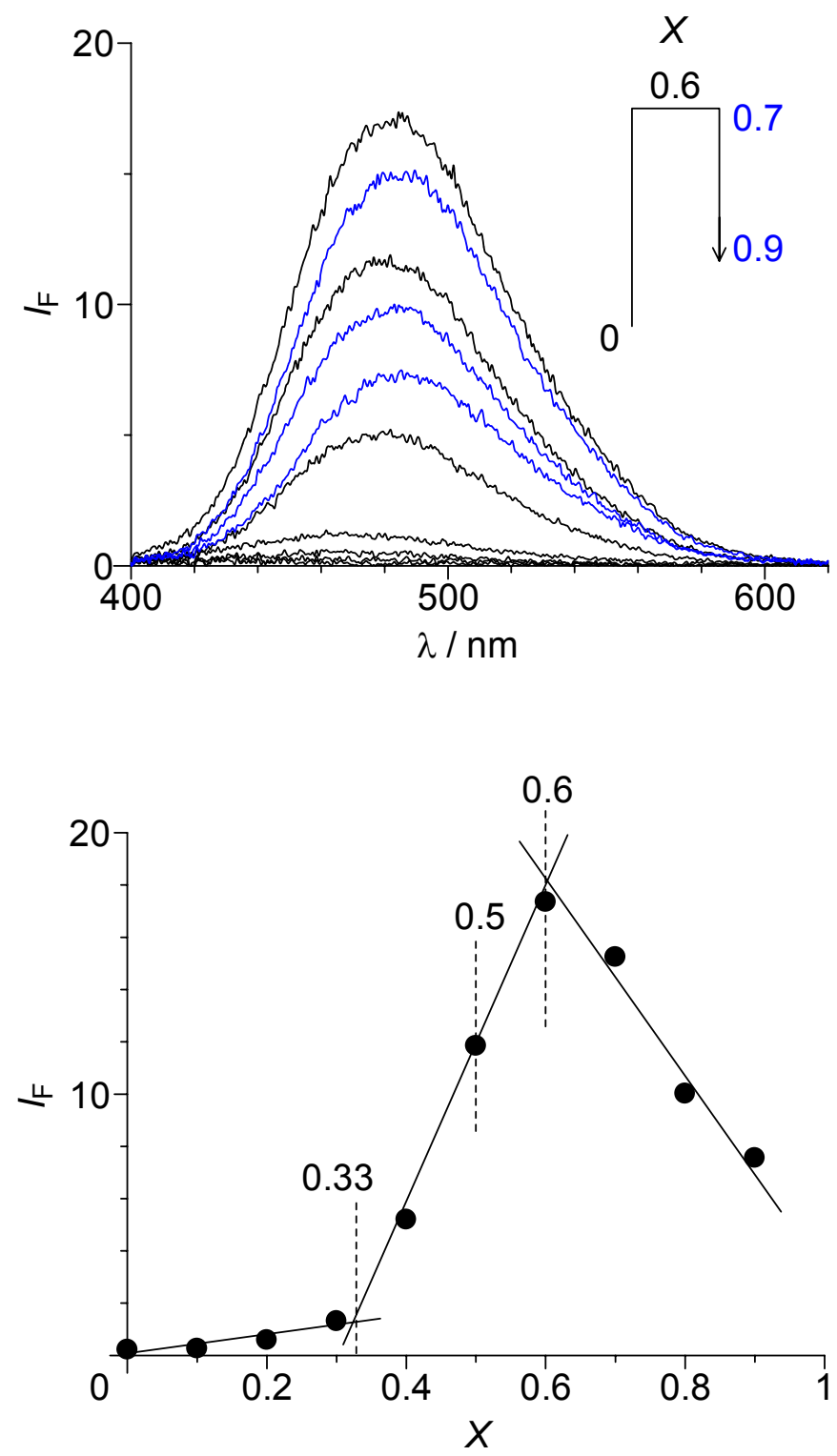

Figure S5. Job's plot of $\mathbf{L}$ with $\mathrm{Hg}^{2+}$ obtained by fluorescence measurements $\left(X=\left[\mathrm{Hg}^{2+}\right] /\left(\left[\mathrm{Hg}^{2+}\right]+\right.\right.$ $\left.[\mathbf{L}] ; \lambda_{\mathrm{em}}=492 \mathrm{~nm}\right)$ ). The total concentration of $\mathbf{L}$ and $\mathrm{Hg}^{2+}$ is $100 \mu \mathrm{M}$. 

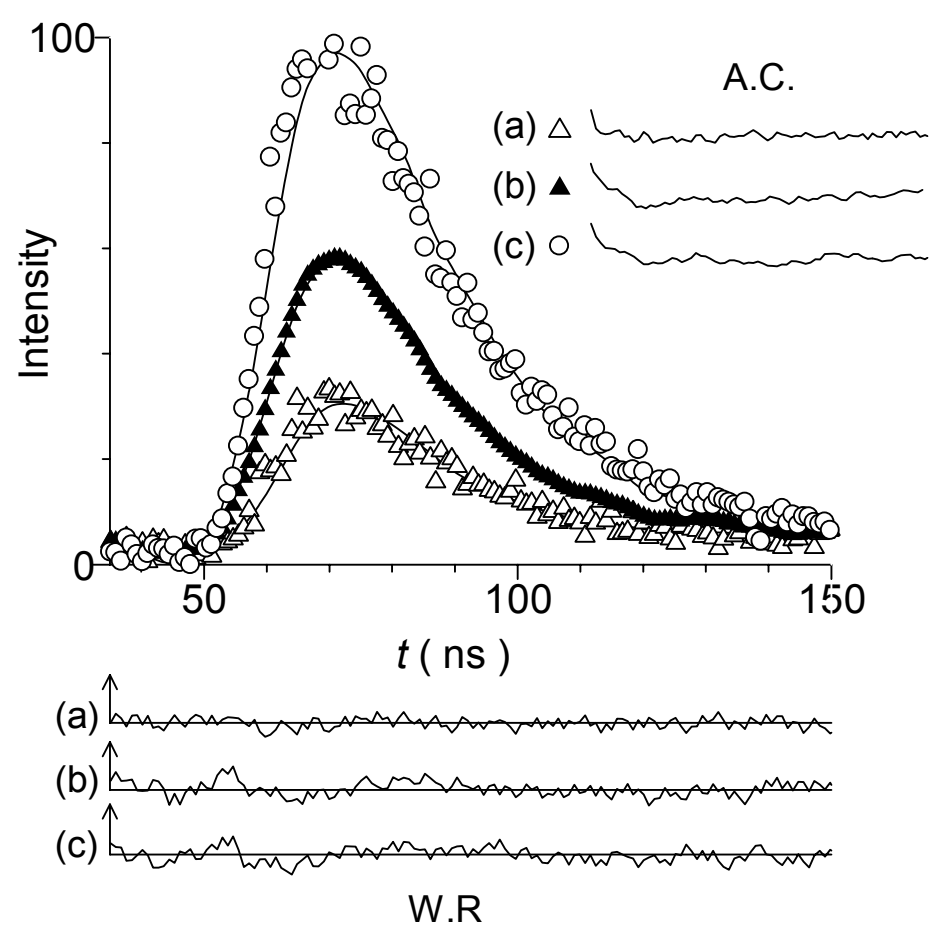

\begin{tabular}{cccc}
\hline & $\begin{array}{c}\left.\mathbf{L}_{2} \mathrm{Hg}_{2}\right]^{4+} \\
\tau / \mathrm{ns}\end{array}$ & $\begin{array}{c}{\left[\mathbf{L}_{2} \mathrm{Hg}_{3}\right]^{6+}} \\
\tau / \mathrm{ns} \\
(a / \%)\end{array}$ & $\chi^{2}$ \\
\hline $\left.\mathrm{Hg}^{2+}\right] /[\mathbf{L}]$ & 7.86 & & 1.29 \\
1 & $(100)$ & 1.38 & 1.99 \\
1.25 & 7.86 & $(42.2)$ & \\
& $(57.8)$ & 11.38 & 2.32 \\
\hline
\end{tabular}

Figure S6. Fluorescence decay profiles $\left(\lambda_{\mathrm{exc}}=358 \mathrm{~nm} ; \lambda_{\mathrm{em}}=480 \mathrm{~nm}\right)$ of $\mathbf{L}$ in acetonitrile measured with (a) 1.0 , (b) 1.25 , and (c) 1.5 equiv of $\mathrm{Hg}^{2+}$, and lifetimes $(\tau)$ and preexponential factors $(a)$ for the respective emitting components. For judging the quality of the fit, autocorrelation (A.C) and weighted residuals (W.R) are also shown in the figure. 

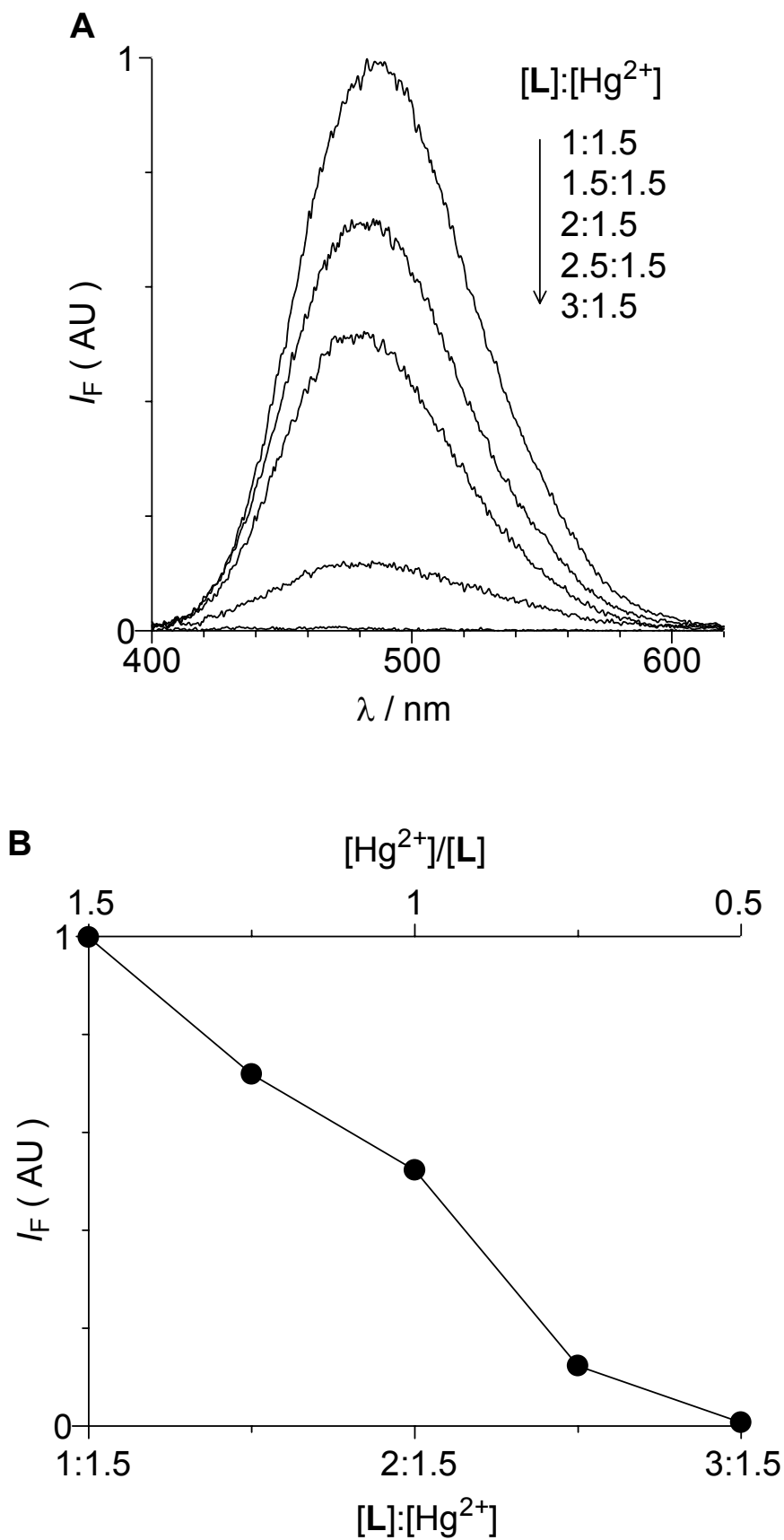

Figure S7. Change in emission spectra and the intensity (monitored at $492 \mathrm{~nm}$ ) of $\mathbf{L}$ in acetonitrile containing 2:3 $\mathbf{L}-\mathrm{Hg}^{2+}$ complex $\left([\mathbf{L}]:\left[\mathrm{Hg}^{2+}\right]=1: 1.5\right)$ upon addition of $\mathbf{L}$. 


\section{Cartesian Coordinates (in $\AA$ ) of 2:1 complex $\left[\mathrm{L}_{2} \mathrm{Hg}\right]^{2+}$.}

\begin{tabular}{|c|c|c|c|c|c|c|c|}
\hline C & 0.374472 & 2.521005 & -2.620367 & C & -4.279856 & 2.112414 & 1.596803 \\
\hline $\mathrm{C}$ & -0.186157 & 1.504452 & -3.648165 & $\mathrm{C}$ & -5.685426 & -1.215157 & 0.413408 \\
\hline $\mathrm{N}$ & 0.281603 & 2.015642 & -1.242111 & $\mathrm{C}$ & -6.834840 & -1.969724 & 0.565041 \\
\hline & 0.194300 & 0.137051 & -3.283742 & $\mathrm{C}$ & -7.903315 & -1.501087 & 1.388959 \\
\hline & 0.550533 & 2.775791 & -0.217032 & $\mathrm{C}$ & -7.789417 & -0.284622 & 2.034906 \\
\hline & 0.659468 & -0.720633 & -4.149137 & $\mathrm{Hg}$ & -0.297777 & -0.268617 & -1.078553 \\
\hline & 0.911139 & 4.217142 & -0.191774 & $\mathrm{H}$ & -0.197715 & 3.474036 & -2.746692 \\
\hline & 1.057904 & -2.090489 & -3.759596 & $\mathrm{H}$ & 1.442370 & 2.749095 & -2.867901 \\
\hline & 0.396546 & -2.841082 & -2.708078 & $\mathrm{H}$ & 0.178558 & 1.801421 & -4.663182 \\
\hline & 0.940251 & -4.123932 & -2.343069 & $\mathrm{H}$ & -1.305302 & 1.556087 & -3.689280 \\
\hline & 2.088413 & -4.640785 & -3.041319 & $\mathrm{H}$ & 0.483300 & 2.311875 & 0.783004 \\
\hline & 2.664812 & -3.931038 & -4.079234 & $\mathrm{H}$ & 0.804775 & -0.454446 & -5.211177 \\
\hline & 2.145203 & -2.656932 & -4.439401 & $\mathrm{H}$ & 2.499543 & -5.610946 & -2.752561 \\
\hline & -0.838182 & -2.401609 & -2.050235 & $\mathrm{H}$ & 3.518190 & -4.333580 & -4.628039 \\
\hline & -1.448582 & -3.242629 & -1.093074 & $\mathrm{H}$ & 2.634250 & -2.122317 & -5.258388 \\
\hline & -0.851686 & -4.466605 & -0.689000 & $\mathrm{H}$ & -1.534237 & -1.725360 & -2.600185 \\
\hline & 0.305418 & -4.905789 & -1.314342 & $\mathrm{H}$ & -2.436336 & -2.992686 & -0.695792 \\
\hline & 2.291820 & 4.648382 & -0.119598 & $\mathrm{H}$ & -1.334260 & -5.073120 & 0.079258 \\
\hline & 2.569477 & 6.057155 & -0.044242 & $\mathrm{H}$ & 0.733161 & -5.873003 & -1.037658 \\
\hline & 1.479048 & 6.993850 & -0.031002 & $\mathrm{H}$ & 1.691345 & 8.063705 & 0.026503 \\
\hline & 0.167793 & 6.554688 & -0.086096 & $\mathrm{H}$ & -0.660522 & 7.264321 & -0.069673 \\
\hline & -0.114247 & 5.162676 & -0.163553 & $\mathrm{H}$ & -1.162165 & 4.855747 & -0.197832 \\
\hline & 3.411448 & 3.743238 & -0.114452 & $\mathrm{H}$ & 3.240192 & 2.667182 & -0.162326 \\
\hline & 4.714330 & 4.201863 & -0.046419 & $\mathrm{H}$ & 5.550148 & 3.500276 & -0.043091 \\
\hline & 4.984771 & 5.602651 & 0.021279 & $\mathrm{H}$ & 6.019403 & 5.944210 & 0.072581 \\
\hline & 3.937677 & 6.504594 & 0.022485 & $\mathrm{H}$ & 4.143530 & 7.575573 & 0.075891 \\
\hline & -1.573248 & -0.138213 & 1.760449 & $\mathrm{H}$ & -1.321809 & 0.859414 & 2.202926 \\
\hline & -0.342771 & -1.084971 & 1.888667 & $\mathrm{H}$ & -2.380957 & -0.577151 & 2.399509 \\
\hline & -2.032869 & 0.049931 & 0.375641 & $\mathrm{H}$ & -0.665950 & -2.152290 & 1.782990 \\
\hline & 0.705816 & -0.832632 & 0.888586 & $\mathrm{H}$ & 0.030431 & -0.978568 & 2.937590 \\
\hline & -3.224214 & 0.477235 & 0.052011 & $\mathrm{H}$ & -3.433342 & 0.565283 & -1.031699 \\
\hline & 1.988657 & -0.886603 & 1.119136 & $\mathrm{H}$ & 2.649816 & -0.728484 & 0.245178 \\
\hline & -4.340545 & 0.881423 & 0.940811 & $\mathrm{H}$ & 4.702593 & -1.455513 & 5.769953 \\
\hline & 2.695545 & -1.099724 & 2.407279 & $\mathrm{H}$ & 3.712583 & 0.731676 & 5.125072 \\
\hline & 3.274475 & -2.383509 & 2.745460 & $\mathrm{H}$ & 2.463131 & 0.970217 & 3.017809 \\
\hline & 4.002721 & -2.507450 & 3.979246 & $\mathrm{H}$ & 2.630844 & -3.490367 & 0.962581 \\
\hline & 4.147719 & -1.360970 & 4.833858 & $\mathrm{H}$ & 3.646159 & -5.631373 & 1.627215 \\
\hline & 3.596900 & -0.141102 & 4.480932 & $\mathrm{H}$ & 4.892965 & -5.838240 & 3.773558 \\
\hline & 2.872273 & -0.012132 & 3.264343 & $\mathrm{H}$ & 5.131556 & -3.868618 & 5.271883 \\
\hline & 3.166267 & -3.550898 & 1.910530 & $\mathrm{H}$ & -7.314088 & 2.153500 & 3.178109 \\
\hline & 3.735464 & -4.757674 & 2.274736 & $\mathrm{H}$ & -5.269630 & 3.539845 & 2.901550 \\
\hline & 4.452196 & -4.877458 & 3.504202 & $\mathrm{H}$ & -3.407388 & 2.761529 & 1.494322 \\
\hline & 4.581344 & -3.778990 & 4.333012 & $\mathrm{H}$ & -4.890320 & -1.600599 & -0.225576 \\
\hline & -5.525537 & 0.056708 & 1.067960 & $\mathrm{H}$ & -6.939156 & -2.929733 & 0.056674 \\
\hline & -6.606423 & 0.526042 & 1.892460 & $\mathrm{H}$ & -8.800967 & -2.111499 & 1.497131 \\
\hline & -6.491685 & 1.796449 & 2.554092 & $\mathrm{H}$ & -8.606270 & 0.075119 & 2.663896 \\
\hline & -5.354327 & 2.571915 & 2.405698 & & & & \\
\hline
\end{tabular}




\section{Cartesian Coordinates (in $\AA$ ) of 2:2 complex $\left[\mathrm{L}_{2} \mathrm{Hg}_{2}\right]^{4+}$.}

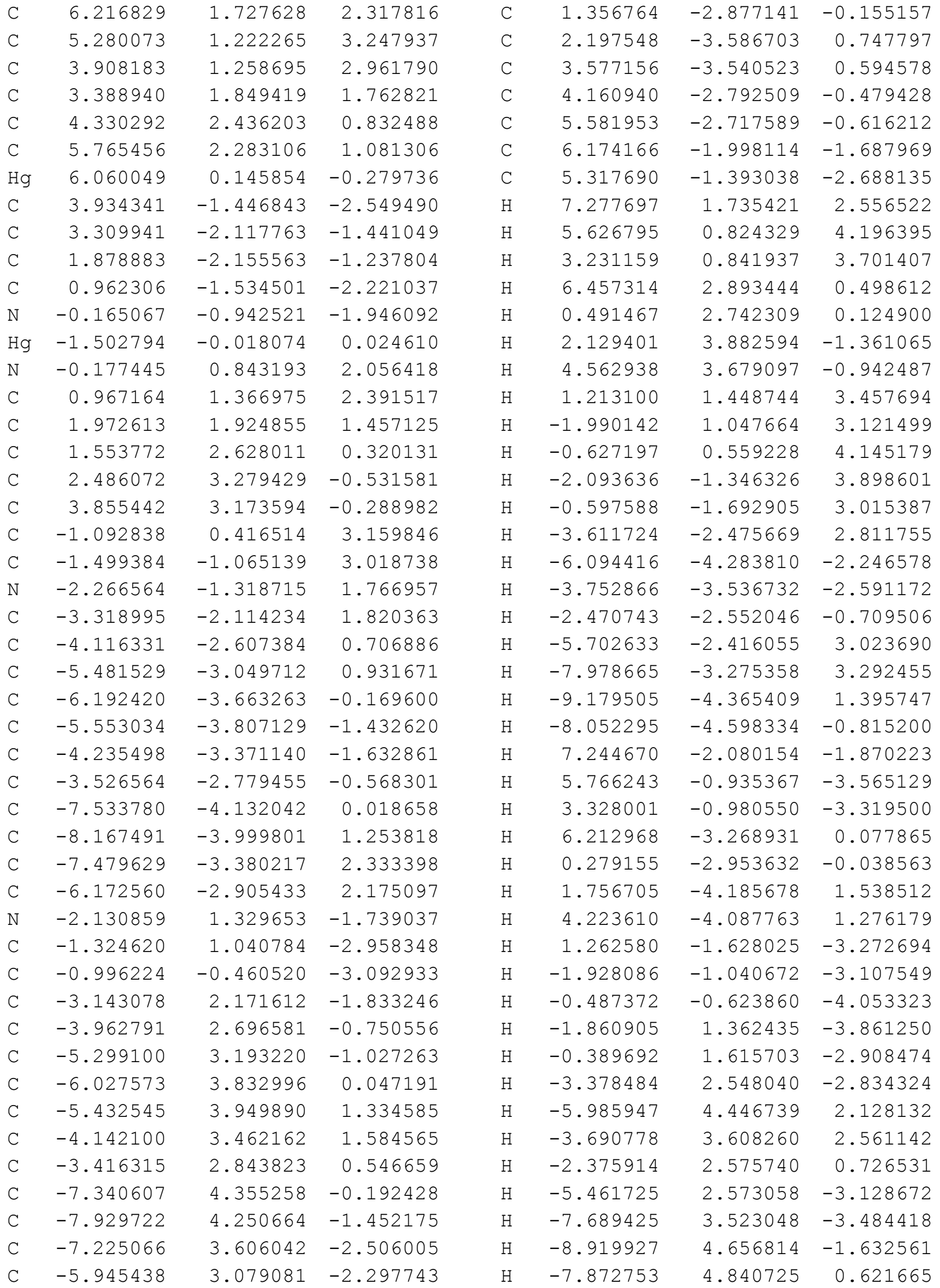


Cartesian Coordinates (in $\AA$ ) of $2: 3$ complex $\left[\mathrm{L}_{2} \mathrm{Hg}_{3}\right]^{6+}$.

\begin{tabular}{|c|c|c|c|c|c|c|c|}
\hline 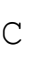 & -7.159483 & 1.790519 & -2.251710 & C & 5.439985 & 1.398433 & 1.823045 \\
\hline & -6.113350 & 1.522287 & -3.152159 & $\mathrm{C}$ & 6.863732 & 1.486529 & 1.659675 \\
\hline & -4.793144 & 1.595339 & -2.724531 & $\mathrm{C}$ & 7.438247 & 2.618922 & 0.979155 \\
\hline & -4.424721 & 1.954263 & -1.392348 & $\mathrm{C}$ & 6.603926 & 3.594392 & 0.474289 \\
\hline & -5.476250 & 2.267045 & -0.463867 & $\mathrm{Hg}$ & 0.154375 & 0.101238 & 0.159743 \\
\hline & -6.865751 & 2.115972 & -0.890538 & $\mathrm{Hg}$ & 6.626666 & -0.015901 & -0.159315 \\
\hline & -3.056519 & 2.053201 & -0.938496 & $\mathrm{Hg}$ & -7.309437 & -0.094773 & -0.152760 \\
\hline & -2.804829 & 2.516901 & 0.350815 & $\mathrm{H}$ & -8.191988 & 1.814005 & -2.598117 \\
\hline & -3.847314 & 2.898697 & 1.220242 & $\mathrm{H}$ & -6.340212 & 1.294869 & -4.189894 \\
\hline & -5.165522 & 2.766152 & 0.824811 & $\mathrm{H}$ & -4.019988 & 1.386573 & -3.458285 \\
\hline & -1.922896 & 1.859264 & -1.862987 & $\mathrm{H}$ & -7.637170 & 2.631186 & -0.310595 \\
\hline & -0.768934 & 1.347126 & -1.587154 & $\mathrm{H}$ & -1.778539 & 2.678198 & 0.667141 \\
\hline & 0.297747 & 1.430858 & -2.611844 & $\mathrm{H}$ & -3.613766 & 3.331208 & 2.188643 \\
\hline & 0.958224 & 0.076319 & -2.888254 & $\mathrm{H}$ & -5.966990 & 3.080677 & 1.489735 \\
\hline & 1.588536 & -0.538863 & -1.694876 & $\mathrm{H}$ & -2.059332 & 2.254067 & -2.875580 \\
\hline & 2.725152 & -1.130798 & -1.885450 & $\mathrm{H}$ & 1.043456 & 2.148316 & -2.247061 \\
\hline & 3.422046 & -2.088455 & -1.008292 & $\mathrm{H}$ & -0.098817 & 1.822392 & -3.555713 \\
\hline & 4.837461 & -2.330574 & -1.126004 & $\mathrm{H}$ & 1.689542 & 0.210041 & -3.694565 \\
\hline & 5.388282 & -3.516990 & -0.531570 & $\mathrm{H}$ & 0.203846 & -0.632136 & -3.254016 \\
\hline & 4.559712 & -4.382845 & 0.232123 & $\mathrm{H}$ & 3.185787 & -1.000035 & -2.872246 \\
\hline & 3.215570 & -4.100849 & 0.393534 & $\mathrm{H}$ & 4.987082 & -5.285047 & 0.662431 \\
\hline & 2.657372 & -2.980052 & -0.244848 & $\mathrm{H}$ & 2.582494 & -4.783187 & 0.952276 \\
\hline & 5.770376 & -1.460455 & -1.833426 & $\mathrm{H}$ & 1.579145 & -2.851530 & -0.222069 \\
\hline & 7.124132 & -1.899031 & -2.078781 & $\mathrm{H}$ & 5.379245 & -0.700368 & -2.511473 \\
\hline & 7.612385 & -3.089855 & -1.523905 & $\mathrm{H}$ & 7.748808 & -1.312233 & -2.751612 \\
\hline & 6.754754 & -3.871762 & -0.760370 & $\mathrm{H}$ & 8.632467 & -3.409245 & -1.718897 \\
\hline & -7.252803 & -1.975616 & 1.958061 & $\mathrm{H}$ & 7.120961 & -4.805925 & -0.338758 \\
\hline & -6.298503 & -1.664137 & 2.942165 & $\mathrm{H}$ & -8.308479 & -2.046317 & 2.217164 \\
\hline & -4.945205 & -1.675367 & 2.625667 & $\mathrm{H}$ & -6.620648 & -1.451682 & 3.957649 \\
\hline & -4.451202 & -2.011932 & 1.328548 & $\mathrm{H}$ & -4.246334 & -1.435167 & 3.421748 \\
\hline & -5.406681 & -2.369876 & 0.316364 & $\mathrm{H}$ & -7.528558 & -2.831738 & -0.015226 \\
\hline & -6.832402 & -2.282628 & 0.625642 & $\mathrm{H}$ & -1.612698 & -2.611182 & -0.501971 \\
\hline & -3.047121 & -2.047926 & 0.990077 & $\mathrm{H}$ & -3.283993 & -3.346260 & -2.168740 \\
\hline & -2.668084 & -2.498034 & -0.272461 & $\mathrm{H}$ & -5.696137 & -3.201413 & -1.669401 \\
\hline & -3.616502 & -2.925473 & -1.224425 & $\mathrm{H}$ & -2.208751 & -2.201308 & 3.004684 \\
\hline & -4.967689 & -2.852349 & -0.940765 & $\mathrm{H}$ & 0.924840 & -1.956026 & 2.644985 \\
\hline & -2.004570 & -1.801883 & 2.005312 & $\mathrm{H}$ & -0.339655 & -1.680360 & 3.847193 \\
\hline & -0.856397 & -1.237957 & 1.823185 & $\mathrm{H}$ & 1.387573 & 0.010376 & 4.107585 \\
\hline & 0.118463 & -1.272406 & 2.938856 & $\mathrm{H}$ & -0.111928 & 0.781208 & 3.585725 \\
\hline & 0.694387 & 0.110099 & 3.263874 & $\mathrm{H}$ & 2.912680 & 1.288553 & 3.317765 \\
\hline & 1.356595 & 0.753144 & 2.104989 & $\mathrm{H}$ & 4.819076 & 5.391235 & -0.408564 \\
\hline & 2.464099 & 1.382661 & 2.320438 & $\mathrm{H}$ & 2.363454 & 5.255049 & -0.196969 \\
\hline & 3.137125 & 2.332557 & 1.412450 & $\mathrm{H}$ & 1.308545 & 3.368924 & 1.007353 \\
\hline & 4.580166 & 2.386527 & 1.293221 & $\mathrm{H}$ & 5.048498 & 0.590412 & 2.437168 \\
\hline & 5.184893 & 3.505856 & 0.604786 & $\mathrm{H}$ & 7.501555 & 0.877903 & 2.305893 \\
\hline & 4.357464 & 4.532474 & 0.071664 & $\mathrm{H}$ & 8.519192 & 2.718314 & 0.918886 \\
\hline & 2.986382 & 4.451481 & 0.184927 & $\mathrm{H}$ & 7.030385 & 4.468333 & -0.012240 \\
\hline & 2.383199 & 5422 & 0.850878 & & & & \\
\hline
\end{tabular}

